\title{
Rashba-Dirac cones at the tungsten surface: Insights from a tight-binding model and thin film subband structure
}

\author{
George Kirczenow ${ }^{1}$ \\ ${ }^{1}$ Department of Physics, Simon Fraser University, Burnaby, British Columbia, Canada V5A $1 S 6$
}

(Dated: November 13, 2018)

\author{
Phys. Rev. B94, 205414 (2016). Published Nov. 10, 2016. \\ URL: http://link.aps.org/doi/10.1103/PhysRevB.94.205414 \\ DOI: 10.1103/PhysRevB.94.205414
}

\begin{abstract}
A tight-binding model of bcc tungsten that includes spin-orbit coupling is developed and applied to the surface states of (110) tungsten thin films. The model describes accurately the anisotropic Dirac cone-like dispersion and Rashba-like spin polarization of the surface states, including the crucial effect of the relaxation of the surface atomic layer of the tungsten towards the bulk. It is shown that the surface relaxation affects the tungsten surface states because it results in increased overlaps between atomic orbitals of the surface atomic layer and nearby layers whereas electric fields that are due to charge transfer between the tungsten and the vacuum near the surface or between the bulk and surface layers do not significantly affect the Rashba-Dirac surface states. It is found that hybridization with bulk modes has differing strengths for thin film surface states belonging to the upper and lower Rashba-Dirac cones and results in reversal of the directions of travel of spin $\uparrow$ and $\downarrow$ electrons in most of the upper Rashba-Dirac cone relative to those expected from phenomenology. It is also shown that intrasite (not intersite) matrix elements of the spin-orbit Hamiltonian are primarily responsible for the formation of the Rashba-Dirac cones, and their spin polarization. This finding should be considered when modeling topological insulators, the spin Hall effect and related phenomena.
\end{abstract}

PACS numbers: 75.70.Tj, 73.20.-r, 71.20.Be, 85.75.-d

\section{INTRODUCTION}

Spin-orbit coupling in non-magnetic materials gives rise to spin-split electronic states in Rashba systems ${ }^{1,2}$ and topological insulators. ${ }^{3,4}$ The resulting locking of spin to the crystal-momentum offers potential avenues for the realization of novel spintronic devices and quantum computation. Electronic states that are spin-split due to strong spin-orbit coupling also occur at the surfaces of some non-magnetic metals, including gold, ${ }^{5-9}$ tungsten, ${ }^{10-23}$ silver, ${ }^{6,9}$ copper, ${ }^{24,25}$ bismuth, ${ }^{26-31}$ antimony, ${ }^{32}$ and iridium. ${ }^{33}$

Following the recent experimental observation at the tungsten (110) surface of Rashba-like spin-split and spin-polarized electronic states forming an anisotropic Dirac cone-like band structure, ${ }^{13}$ there has been renewed interest in developing a better understanding of this system. ${ }^{13-23}$ It has been demonstrated ${ }^{14}$ that the experimentally observed anisotropic Dirac cone-like band structure can be fitted accurately by a phenomenological third order Rashba model for surfaces with $C_{2 v}$ symmetry. ${ }^{34} A b$ initio calculations ${ }^{15,17,19,20,22,23}$ have also accounted for the experimental data, including the Dirac conelike dispersion and the spin polarizations of the observed surface states. However, it is also of interest to investigate the underlying physics with the help of a tight-binding model that, unlike the anisotropic Rashba phenomenology, ${ }^{14}$ is atomistic and also can provide insights that are complimentary to those obtained from ab initio calculations. ${ }^{15,17,19,20,22,23}$ Such a tightbinding model is introduced here and applied to tungsten thin films with (110) surfaces and their electronic subband structure.

The present tight-binding model is a modification of extended Hückel theory, ${ }^{35-38}$ generalized to include spin-orbit coupling as was proposed in Refs. 39 and 40 for molecular nanomagnets. It is parameterized to accurately reproduce the electronic band structure of bulk b.c.c. tungsten, both with ${ }^{41,42}$ and without ${ }^{43}$ the inclusion of spin-orbit coupling. The results obtained for the W(110) Rashba-Dirac cone surface states are in remarkably good agreement with those of the previous $a b$ initio $^{15,17,19,20,23}$ calculations for semi-infinite tungsten crystals. However, the present tight-binding approach and investigation of the thin film subband structure yields additional insights outlined below.

Although the present model is parameterized by fitting to only the bulk electronic structure of tungsten, it also captures correctly the important effect ${ }^{17}$ on the Rashba-Dirac cone surface states of the relaxation of the $\mathrm{W}(110)$ surface atomic layer towards the bulk that is observed experimentally. ${ }^{44,45}$ It is shown below that this effect is due to the increased overlaps between atomic orbitals of the tungsten surface and bulk atomic layers induced by the relaxation. It is also shown that the contribution of electric fields that are due to charge transfer between the tungsten and the vacuum near the surface or between the bulk and surface layers to this effect and their influence on the Rashba-Dirac cone-like dispersion of tungsten (110) surface states are both at most minor.

The present work also reveals that hybridization with bulk modes results in the directions of travel of spin $\uparrow$ and $\downarrow$ electrons in the thin film's surface states belonging to most of the upper Rashba-Dirac cone being reversed relative to those predicted by phenomenological Rashba models. This reversal is due to dense anticrossings between the surface and bulklike states that arise from their strong hybridization. It is also shown that hybridization between the bulk-like states and lower Rashba-Dirac cone states is much weaker than that be- 
tween the bulk states and upper Rashba-Dirac cone states, suggesting that the intrinsic lifetimes of the upper and lower Rashba-Dirac cone surface states may differ significantly.

Finally, a comparison of the present model with the standard tight binding formulation ${ }^{4,46}$ of the original Rashba model $^{1}$ is made and strongly suggests that the mechanism responsible for the Rashba-like dispersion and spin polarization of W(110) surface states differs fundamentally from that of the original Rashba effect in nearly free 2DEG's at semiconductor interfaces. This conclusion is consistent with that reached previously by entirely different reasoning ${ }^{47}$ for surface states of other materials. It may be expected to have significant implications for theoretical work on topological insulators, spin Hall phenomena and related topics.

The remainder of this article is organized as follows: The tight-binding model of tungsten is explained in Section II. The results obtained by applying the model to tungsten (110) thin films are presented and discussed in Section III. The conclusions drawn from this work are summarized in Section IV.

\section{MODEL}

The tight binding model developed here is based on extended Hückel theory, ${ }^{35-38}$ a semi-empirical tight binding scheme from quantum chemistry that is formulated in terms of a small set of Slater-type atomic valence orbitals $\left\{\left|\phi_{i}\right\rangle\right\}$, their overlaps $O_{i j}=\left\langle\phi_{i} \mid \phi_{j}\right\rangle$ and a Hamiltonian matrix $H_{i j}^{0}=$ $\left\langle\phi_{i}\left|H^{0}\right| \phi_{j}\right\rangle$. In extended Hückel theory the diagonal Hamiltonian elements $H_{i i}^{0}=\epsilon_{i}$ are chosen to be the (negative) atomic orbital ionization energies. The Hamiltonian matrix elements for $i \neq j$ are approximated by

$$
H_{i j}^{0}=K_{i j} O_{i j}\left(\epsilon_{i}+\epsilon_{j}\right) / 2
$$

In the Wolfsberg-Helmholz form of extended Hückel theory, ${ }^{35}$ the empirical parameters $K_{i j}$ are all set to 1.75 in order for the model to yield approximate energy levels for a variety of simple molecules. In the present work the $\epsilon_{i}$ and $K_{i j}$ are fitting parameters chosen so that the model accurately reproduces the non-relativistic band structure of bulk bcc tungsten given in Ref. 43 if the Hamiltonian matrix is given by equation (1) and the overlap matrix $O_{i j}$ is calculated using a standard extended Hückel software package. ${ }^{48}$ First, second and third neighbor matrix elements $H_{i j}^{0}$ and $O_{i j}$ are included in the present model. The values of the parameters $\epsilon_{i}$ and $K_{i j}$ used in this work are given in Tables I and II. With these parameter values, the present model (without spin-orbit coupling) matches the non-relativistic band structure of bulk bcc tungsten given in Ref. 43 well throughout the Brillouin zone in the energy range from $\sim 10 \mathrm{eV}$ below the Fermi level to $\sim 10 \mathrm{eV}$ above the Fermi level.

It is worth noting that in the tight-binding model presented in Ref. 43, all of the matrix elements $H_{i j}^{0}$ and $O_{i j}$ are treated as fitting parameters. An advantage of the present methodology is that here, unlike in Ref. 43, the overlap matrix elements $O_{i j}$ are not fixed but depend on the atomic geometry of the tungsten and hence the present formalism can treat deformations of the tungsten crystal whereas the tight-binding model
Table I. Tight-binding orbital energy parameters for bcc tungsten used in the present work. The $x, y$ and $z$ axes are aligned with the cubic crystal axes of bcc tungsten.

\begin{tabular}{c|c|c|c|c} 
Orbital & $6 \mathrm{~s}$ & $6 \mathrm{p}$ & $5 \mathrm{~d}_{x^{2}-y^{2}}, 5 \mathrm{~d}_{z^{2}}$ & $5 \mathrm{~d}_{x y}, 5 \mathrm{~d}_{x z}, 5 \mathrm{~d}_{y z}$ \\
\hline$\epsilon_{i}(\mathrm{eV})$ & -11.907697 & -4.855731 & -11.271223 & -10.365356
\end{tabular}

Table II. Tight-binding parameters $K_{i j}=K_{j i}$ for bcc tungsten. $\alpha, \alpha^{\prime}=x, y$ or $z$. $\beta=x y, x z$ or $y z . \quad \gamma=x^{2}-y^{2}$ or $z^{2}$. $\delta, \delta^{\prime}=x y, x z, y z, x^{2}-y^{2}$ or $z^{2}$. The $x, y$ and $z$ axes are aligned with the cubic crystal axes of bcc tungsten. The tungsten lattice parameter is $a=3.16 \AA$.

\begin{tabular}{l|c|c|c} 
neighbor & first & second & third \\
\hline$K_{s, s}$ & 2.15 & 1.50 & 2.25 \\
\hline$K_{p_{\alpha}, p_{\alpha}}$ & 1.75 & 3.00 & 3.00 \\
\hline$K_{p_{\alpha}, p_{\alpha^{\prime}}, \alpha \neq \alpha^{\prime}}$ & 2.30 & 2.00 & 2.00 \\
\hline$K_{d_{\beta}, d_{\beta}}$ & 2.30 & 1.70 & 1.70 \\
\hline$K_{d_{\gamma}, d_{\gamma}}$ & 2.00 & 2.00 & 2.00 \\
\hline$K_{d_{\delta}, d_{\delta^{\prime}}}, \delta \neq \delta^{\prime}$ & 1.95 & 2.00 & 2.00 \\
\hline$K_{s, p_{\alpha}}$ & 2.75 & 2.15 & 2.25 \\
\hline$K_{s, d_{\delta}}$ & 2.25 & 2.30 & 2.40 \\
\hline$K_{p_{\alpha}, d_{\gamma}}$ & 2.40 & 2.00 & 3.50 \\
\hline$K_{p_{\alpha}, d_{\beta}}$ & 1.75 & 2.00 & 2.00
\end{tabular}

in Ref. 43 cannot. This is important since the relaxation of the $\mathrm{W}(110)$ surface atomic layer towards the bulk ${ }^{44,45}$ strongly affects the surface states ${ }^{17}$ that are the topic of the present work.

$H_{i j}^{0}$, like standard extended Hückel theory, does not include spin-orbit coupling which is included in the present tight binding model using the formalism developed in Refs. 39 and 40 to treat spin-orbit coupling in molecular nanomagnets. As shown in Refs. 39 and 40, the spin-orbit Hamiltonian $H_{\text {SO }}=\frac{\hbar}{(2 m c)^{2}} \boldsymbol{\sigma} \cdot \nabla V(\mathbf{r}) \times \mathbf{p}$ can be approximated as a sum of atomic contributions and its tight-binding matrix elements can then be expressed as intrasite and intersite terms. The intrasite matrix elements for site $\alpha$ are ${ }^{39,40}$

$$
\left\langle\Psi_{\alpha l d s}\left|H_{\mathrm{SO}}^{\mathrm{intra}}\right| \Psi_{\alpha l^{\prime} d^{\prime} s^{\prime}}\right\rangle=\frac{\zeta_{l \alpha}}{\hbar^{2}}\left\langle\alpha l d s\left|\mathbf{S} \cdot \mathbf{L}_{\alpha}\right| \alpha l d^{\prime} s^{\prime}\right\rangle \delta_{l l^{\prime}}
$$

where

$$
\zeta_{l \alpha}=\left\langle R_{\alpha l}\left|\frac{1}{2 m^{2} c^{2}} \frac{1}{\left|\mathbf{r}-\mathbf{r}_{\alpha}\right|} \frac{d V_{\alpha}\left(\left|\mathbf{r}-\mathbf{r}_{\alpha}\right|\right)}{d\left(\left|\mathbf{r}-\mathbf{r}_{\alpha}\right|\right)}\right| R_{\alpha l}\right\rangle
$$

and the atomic orbital $\left|\Psi_{\alpha l d s}\right\rangle$ for site $\alpha$ is the product of a radial part $\left|R_{\alpha l}\right\rangle$ and directed atomic orbital $|\alpha l d s\rangle$. Here $l$ is the orbital angular momentum quantum number, $d$ may be $s, p_{x}, p_{y}, p_{z}, d_{x y}, d_{x z}, \ldots$ depending on the value of $l$, and $s$ is the spin quantum number. The principal quantum number $n$ is suppressed. $\mathbf{S}$ is the spin angular momentum, $\mathbf{L}_{\alpha}$ is the orbital angular momentum relative to the nucleus located at $\mathbf{r}_{\alpha}$ and $V_{\alpha}$ is the electron potential energy contribution of atom $\alpha$. 
The intersite matrix elements are ${ }^{39,40}$

$$
\begin{array}{r}
\left\langle\Psi_{\alpha^{\prime} l^{\prime} d^{\prime} s^{\prime}}\left|H_{\mathrm{SO}}^{\mathrm{inter}}\right| \Psi_{\alpha l d s}\right\rangle=\left(1-\delta_{\alpha^{\prime} \alpha}\right) \times \\
\sum_{d^{\prime \prime} s^{\prime \prime}}\left[O_{\alpha^{\prime} l^{\prime} d^{\prime} s^{\prime}, \alpha l d^{\prime \prime} s^{\prime \prime}}\left\langle\Psi_{\alpha l d^{\prime \prime} s^{\prime \prime}}\left|H_{\mathrm{SO}}^{\mathrm{intra}}\right| \Psi_{\alpha l d s}\right\rangle\right. \\
\left.+O_{\alpha l d s, \alpha^{\prime} l^{\prime} d^{\prime \prime} s^{\prime \prime}}\left\langle\Psi_{\alpha^{\prime} l^{\prime} d^{\prime \prime} s^{\prime \prime}}\left|H_{\mathrm{SO}}^{\mathrm{intra}}\right| \Psi_{\alpha^{\prime} l^{\prime} d^{\prime} s^{\prime}}\right\rangle^{*}\right]
\end{array}
$$

where $O_{\alpha l d s, \alpha^{\prime} l^{\prime} d^{\prime} s^{\prime}}=\left\langle\Psi_{\alpha l d s} \mid \Psi_{\alpha^{\prime} l^{\prime} d^{\prime} s^{\prime}}\right\rangle$ is the overlap between the valence orbitals on sites $\alpha$ and $\alpha^{\prime}$.

The matrix elements of $\mathbf{S} \cdot \mathbf{L} / \hbar^{2}$ that appear in Eq. (2) have been evaluated analytically. Explicit expressions for them in the cubic harmonics representation for the angular parts of $s, p$ and $d$ orbitals that is used in the YAEHMOP extended Hückel software package ${ }^{48}$ are given in Table II of Ref. 49. They do not depend on which material is being considered since only the angular parts of the atomic orbitals are involved; the effects of the radial parts are included in the parameters $\zeta_{l \alpha}$ defined by Eq. (3). The parameters $\zeta_{l \alpha}$ in Eq. (2) and (3) characterize the strength of the spin-orbit coupling. In the present work their values are determined by fitting the bulk tungsten band structure obtained for the complete tight-binding Hamiltonian $H=H^{0}+H_{\mathrm{SO}}^{\text {intra }}+H_{\mathrm{SO}}^{\text {inter }}$ (with $H^{0}$ parameterized according to Tables I and II) to the relativistic $a b$ initio band structures for bulk tungsten in Refs. 41 and 42 . This yields $\zeta_{5 d \alpha}=0.311 \mathrm{eV}$ and $\zeta_{6 p \alpha}=1.74 \mathrm{eV}$. These values were obtained by fitting to the $a b$ initio values ${ }^{41,42}$ of band splittings opened by the spin-orbit coupling at the $\Gamma$ point (specifically, the gap centered $\sim 1 \mathrm{eV}$ below the Fermi level) and $\mathrm{H}$ point (the gap centered $\sim 8.5 \mathrm{eV}$ above the Fermi level) of the Brillouin zone. The size of the former gap is affected mainly by $\zeta_{5 d \alpha}$ and the latter mainly by $\zeta_{6 p \alpha}$. With these parameter values, the band structure obtained from the present model (with spin-orbit coupling) matches the relativistic band structures of bulk bec tungsten given in Refs. 41 and 42 reasonably well throughout the Brillouin zone in the energy range from $\sim 10$ $\mathrm{eV}$ below the Fermi level to $\sim 10 \mathrm{eV}$ above the Fermi level.

\section{RESULTS}

Fig. 1 shows the calculated energies $E$ of the subband Bloch states vs. in-plane wave vector for a 50 atomic layer thin film of tungsten with (110) surfaces for high symmetry cuts $\bar{\Gamma}-\overline{\mathrm{H}}$ and $\bar{\Gamma}-\overline{\mathrm{N}}$ through the $2 \mathrm{D}$ Brillouin zone that is displayed in Fig. 2(a). The surface atomic layers of the film are relaxed towards the bulk by the experimentally measured ${ }^{44,45}$ $2.75 \%$ of the bulk interlayer spacing. Surface states are plotted in color. Those with probabilities $P$ of the electron being in the (110) surface atomic layer are shown in red, orange and yellow for $P>0.3,0.3>P>0.15$ and $0.15>P>0.075$, respectively. The anisotropic upper and lower Rashba-Dirac cones with their common apex at $\bar{\Gamma}$ are clearly visible, superposed in color on the discrete subband structure of the thin film. The energy splittings between the upper and lower cones are much larger in the $\bar{\Gamma}-\overline{\mathrm{H}}$ direction than in the $\bar{\Gamma}-\overline{\mathrm{N}}$ direction, as expected from previous work on the surfaces of macroscopic crystals. ${ }^{13-21,23}$
The strongly spin polarized surface states are shown in Fig. 3 for a cut through the 2D Brillouin zone along the line $\overline{\mathrm{H}}-\bar{\Gamma}-\overline{\mathrm{H}}$ in the Brillouin zone. The states shown in color have a strong presence on the (110) surface atomic layer (as in Fig. 1) and also are strongly spin polarized, i.e., more than $90 \%$ spin $\uparrow$ or spin $\downarrow$ in the (110) surface atomic layer. In Fig. 3, the spin quantization axis is in the (110) plane and perpendicular to the wave vector of the subband Bloch state. As expected for Rashba spin-split states ${ }^{1,2}$ and consistent with experiments and previous theories for (110) surfaces of macroscopic tungsten crystals, ${ }^{13-21,23}$ the spin polarization is opposite for the states of the upper and lower Rashba-Dirac cones with the same in-plane $k$ vector and also opposite for states with the same energy and opposite $k$ vectors. The results for the opposite $(\overline{1} \overline{1} 0)$ surface layer (not shown) are similar but the roles of spin $\uparrow$ and $\downarrow$ are interchanged.

The results shown in Figures 1 and 3 agree very well with the findings of both experiments and $a b$ initio calculations, ${ }^{13-23}$ if one allows for the fact that the previous work has focused on the Rashba-Dirac cone states at the (110) surfaces of macroscopic tungsten crystals that do not have the discrete subband structure of the thin films considered here. However, the thin film character of the present system makes possible new insights into the effects that hybridization with the bulk like states has on the Rashba-Dirac cone surface states. In particular, as is clearly visible in Figures 1 and 3, the strong hybridization with bulk-like states breaks up the upper Rashba-Dirac cone in the $\bar{\Gamma}-\overline{\mathrm{H}}$ direction into a series of anticrossings. This results in the direction of group velocities $v=\frac{1}{\hbar} \frac{\partial E}{\partial k_{\bar{\Gamma}-\bar{H}}}$ of electrons in the states of the upper cone (except extremely close to the cone's apex) being opposite to that predicted by considering only the over all dispersion of the Rashba-Dirac cone without examining in detail the effects of hybridization on individual electronic subband states.

In view of the Rashba-like locking of the spin orientation to the direction of the wave vector of the Rashba-Dirac cone surface states in Fig. 3, this behavior of the group velocity is interesting from the perspective of spintronics. This is because it means that in a thin film the directions of travel of spin $\uparrow$ and $\downarrow$ surface state electrons can be the reverse of the directions predicted from Rashba phenomenology and also from the $E(k)$ dispersion of surface states of semi-infinite crystals deduced from $a b$ initio calculations (such as those in Ref. 17) of spectral densities of states in the Brillouin zone.

On close inspection, a similar reversal relative to the over all dispersion of the Rashba-Dirac cone is found for the group velocities of electrons in states of the upper Rashba-Dirac cone in the $\bar{\Gamma}-\overline{\mathrm{N}}$ direction in Fig. 1. However, hybridization has no such effect on the lower Rashba-Dirac cone in the $\bar{\Gamma}-\overline{\mathrm{H}}$ direction; no anticrossings are visible there in Figures 1 and 3. Also while a series of anticrossings is present in the lower Rashba-Dirac cone in the $\bar{\Gamma}-\overline{\mathrm{N}}$ direction, the hybridization with the bulk states there is extremely weak so that the anticrossings occupy only very narrow ranges of $k$-space. The very different degrees of hybridization of the bulk-like states with the upper and lower Rashba-Dirac cone surface states may be expected to result in different intrinsic lifetimes of electrons in surface states belonging to the upper and lower 


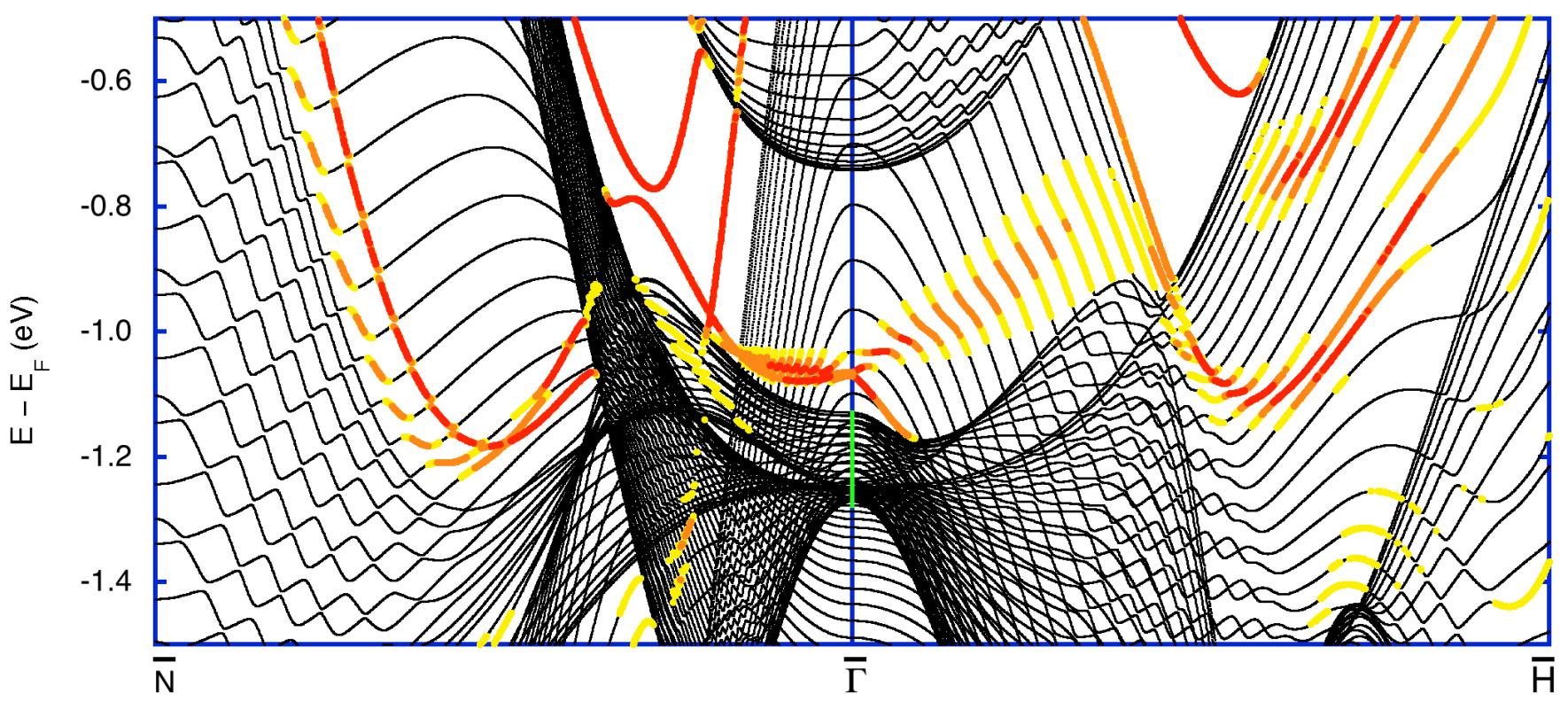

Figure 1. (Color online) Calculated energies $E$ of subband Bloch states vs. in-plane wave vector for a 50 atomic layer thin film of tungsten with (110) surfaces for high symmetry directions in the 2D Brillouin zone. The surface atomic layers of the film are relaxed towards the bulk consistent with experiment. ${ }^{44,45}$ States with larger probabilities $P$ of the electron being in the (110) surface atomic layer are shown in color, $P>0.3$ in red, $0.3>P>0.15$ in orange, $0.15>P>0.075$ in yellow. Results for the opposite (1110) surface layer are similar. The upper and lower anisotropic Rashba-Dirac cones come together at their common apex at the $\bar{\Gamma}$ point. The green line marks the energy range with a high density of bulk-like states at the $\bar{\Gamma}$ point.
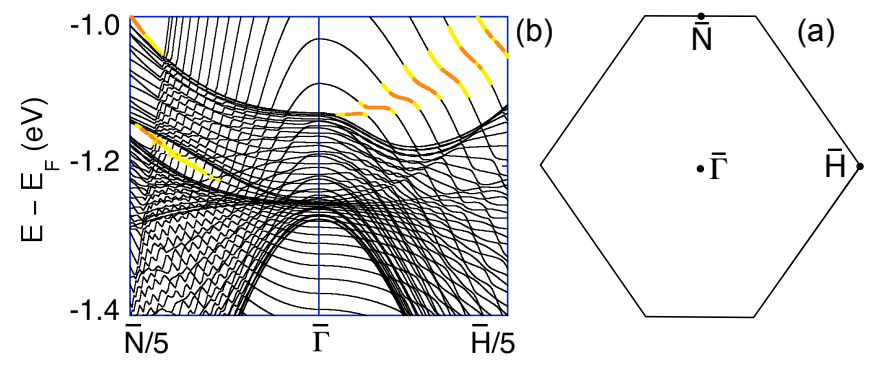

Figure 2. (Color online) (a) Brillouin zone of (110) tungsten film. (b) Dispersion near apex of Rashba-Dirac cone for 50 atomic layer thin film of tungsten with (110) surfaces and no surface relaxation. Colored lines indicate states with larger probabilities of the electron being in the (110) surface atomic layer. Notation as in Fig. 1. Of the Rashba-Dirac cones only a part of the upper cone in the $\bar{\Gamma}-\overline{\mathrm{H}}$ direction (shown in orange and yellow at the upper right) is visible.

cones.

A similar calculation to that for Fig. 1 has been carried out for a film with no surface relaxation, i.e., the bulk structure of bcc tungsten was adopted without modification throughout the 50 atomic layer film. The results are shown in Fig. 2(b) for Bloch state energies and wave vectors near the location of the Rashba-Dirac cone apex of Fig. 1. Only part of the upper branch of the Rashba-Dirac cone in the $\bar{\Gamma}-\overline{\mathrm{H}}$ direction is visible in Fig. 2(b) where it is lower in energy by $\sim 0.1 \mathrm{eV}$ than in Fig. 1. Evidently, the surface relaxation is necessary for the Dirac cone apex, the lower Dirac cone in the $\bar{\Gamma}-\overline{\mathrm{H}}$ direction and both the upper and lower cones in the $\bar{\Gamma}-\overline{\mathrm{N}}$ direction to be present for the tight binding model developed here, as has also been the case for previous $a b$ initio $^{17}$ calculations. In both the present tight binding model and the previous $a b$ initio calculations the surface relaxation opens an energy gap between the apex of the Rashba-Dirac cones and the top of the closest energy range (marked in green in Fig. 1) with an especially high density of bulk states at the $\bar{\Gamma}$ point. In the present model the size of this gap is $\sim 0.06 \mathrm{eV}$ for the relaxed structure, a very similar value to those obtained from $a b$ initio calculations, for example, also $\sim 0.06 \mathrm{eV}$ in Ref. 17. This gap is important since if it were to close or become negative (as in Fig. 2(b) for the unrelaxed surface structure) the states of the RashbaDirac cones that fall in the energy range with the high density of bulk states would hybridize so strongly with the bulk states as to be damped out, as in Fig. 2(b), and would no longer be observable as surface states.

The degree of quantitative agreement between the above results for the Rashba-Dirac surface states and both experiments and $a b$ initio calculations for macroscopic tungsten crystals $^{13-23}$ is quite remarkable since all of the parameters of the tight binding model (described in Section II) have been fitted only to the electronic band structure ${ }^{41-43}$ of bulk bcc tungsten. The only information about the tungsten surface that has been used as input in the present work is structural, namely, the experimentally measured relaxation distance ${ }^{44,45}$ of surface atomic layer towards the bulk. No fitting of any kind to the properties of surface electronic states has been carried out. This makes it possible to draw some previously inaccessible conclusions about the nature of the tungsten (110) RashbaDirac surface states and the mechanism responsible for them, 


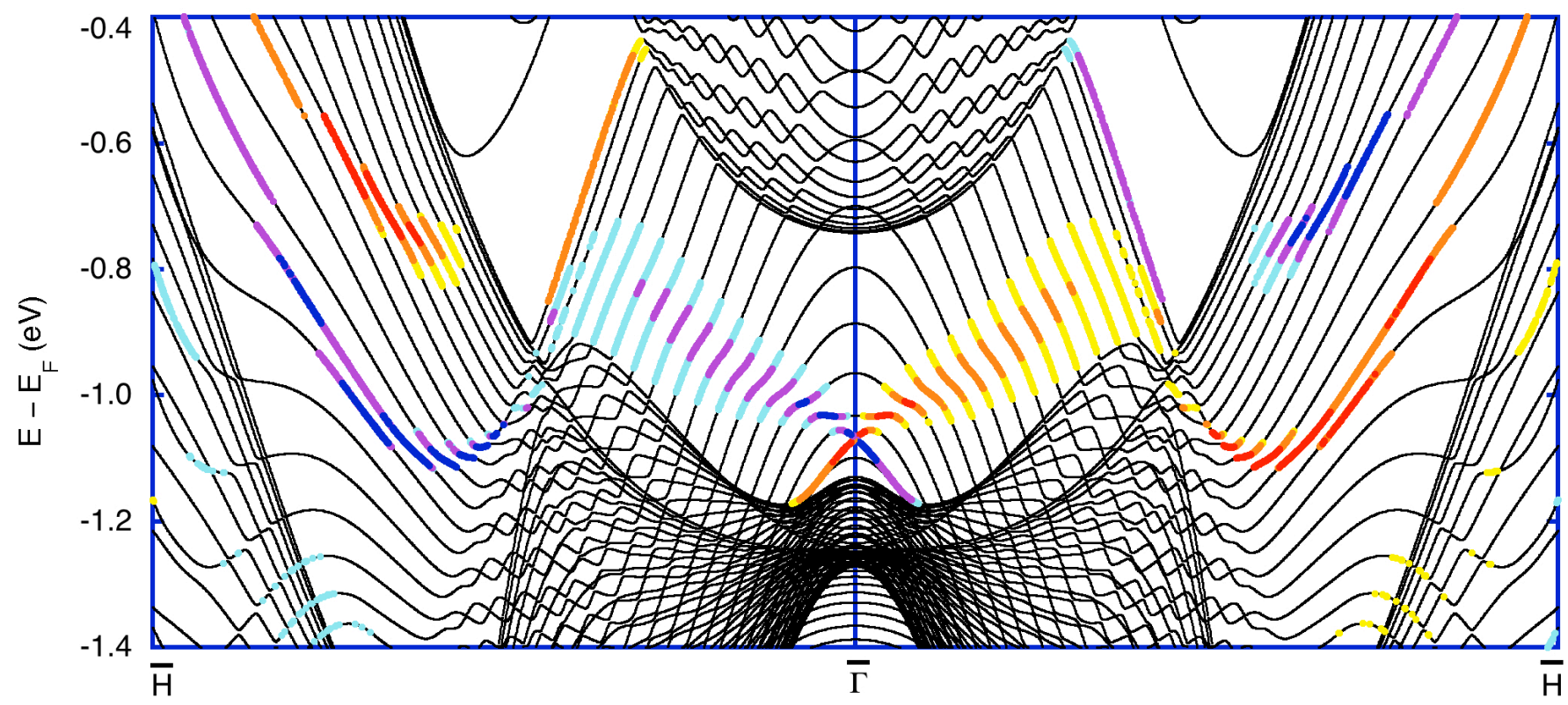

Figure 3. (Color online) Surface states with strong spin polarization (shown in color) in the (110) surface atomic layer of a 50 atomic layer thin film of tungsten with relaxed (110) surfaces. Calculated energies $E$ of subband Bloch states vs. wave vector are plotted for a cut through the 2D Brillouin zone in the $\overline{\mathrm{H}}$ direction. The spin quantization axis is in the (110) plane and perpendicular to the wave vector of the Bloch state. States with more than $90 \%$ spin $\uparrow$ in the (110) surface atomic layer and with probabilities $P$ of the electron being in the (110) surface atomic layer are shown in red, orange and yellow for $P>0.3,0.3>P>0.15$ and $0.15>P>0.075$ respectively. States with more than $90 \%$ spin $\downarrow$ in the (110) surface atomic layer and with probabilities $P$ of the electron being in the (110) surface atomic layer are shown in dark blue, mauve and pale blue for $P>0.3,0.3>P>0.15$ and $0.15>P>0.075$ respectively.

based on what is and is not included in the Hamiltonian of the present tight-binding model.

Importantly, the present tight binding model does not include electric fields that are due to charge transfer between the tungsten and the vacuum near the surface or between the bulk and surface layers, but despite this the Rashba-Dirac cones that it yields agree very well with the results of the ab initio calculations and with experiment. Therefore, although $a b$ initio calculations indicate that such electric fields are present ${ }^{20}$ and they may, in principle, give rise to a Rashba effect, the present work indicates that their effect on the properties of the $\mathrm{W}(110)$ surface states with Dirac cone-like dispersion is at most minor. Similarly, the excellent agreement between the predictions of the present model for the very important upward shift in energy of the Rashba-Dirac surface states due to surface relaxation with the predictions of the $a b$ initio calculations ${ }^{17}$ (as discussed above) indicates that the same electric fields make an at most minor contribution to this effect. The present model shows that this upward shift is mainly due to the increase in the overlaps $O_{i j}$ between atomic orbitals of the surface and bulk atomic layers of the tungsten that results from the surface relaxation and the associated changes in the Hamiltonian matrix elements between these orbitals through Eq. 1.

In the tight-binding formulation ${ }^{46}$ of the original Rashba model, ${ }^{1}$ the only matrix elements of the spin-orbit Hamiltonian $H_{\text {SO }}$ that are considered are those that connect orbitals with opposite spin on neighboring sites. These give rise to the characteristic Rashba dispersion and spin polarization of the electronic states. This intersite-spin-flip tight binding Rashba Hamiltonian was introduced ${ }^{46}$ in work on spin precession in Datta-Das transistors ${ }^{50}$ involving gated nanowires formed from 2-dimensional electron gases (2DEG's) in semiconductors. It has more recently been applied ${ }^{4}$ in studies of the quantum spin Hall effect in topological insulators. The present tight-binding Hamiltonian includes both intrasite [Eq.(2)] and intersite [Eq.(4)] matrix elements of the spin-orbit Hamiltonian. In view of the above it is of interest to investigate the roles that the intrasite and intersite matrix elements of the spin-orbit Hamiltonian play in the formation of the RashbaDirac cones of W(110) surface states. This is addressed in Fig.4 where the results are shown of the same calculation as for Fig. 3 but with the intersite matrix elements of the spinorbit Hamiltonian set to zero. In Fig.4, as in Fig.3, surface states with more than $90 \%$ spin $\uparrow$ or spin $\downarrow$ in the (110) surface atomic layer are shown in color, using the same color scheme as in Fig.3. The Rashba-Dirac cones of strongly spin polarized surface states are still present in Fig.4 and the RashbaDirac cone dispersion is little changed from that in Fig.3. However, for the states of the Rashba-Dirac cones in Fig.4 the probabilities of finding the electron in the surface atomic layer are significantly lower than in the corresponding states in Fig. 3 and the spin polarizations are typically also not quite as strong. For some of the strongly spin polarized surface states not belonging to the Rashba-Dirac cones, the probability for finding the electron in the surface layer is substantially larger (state $a$ ) or smaller (state $b$ ) in Fig.4 than in Fig.3. It follows that intrasite (and not intersite) matrix elements of the tight- 


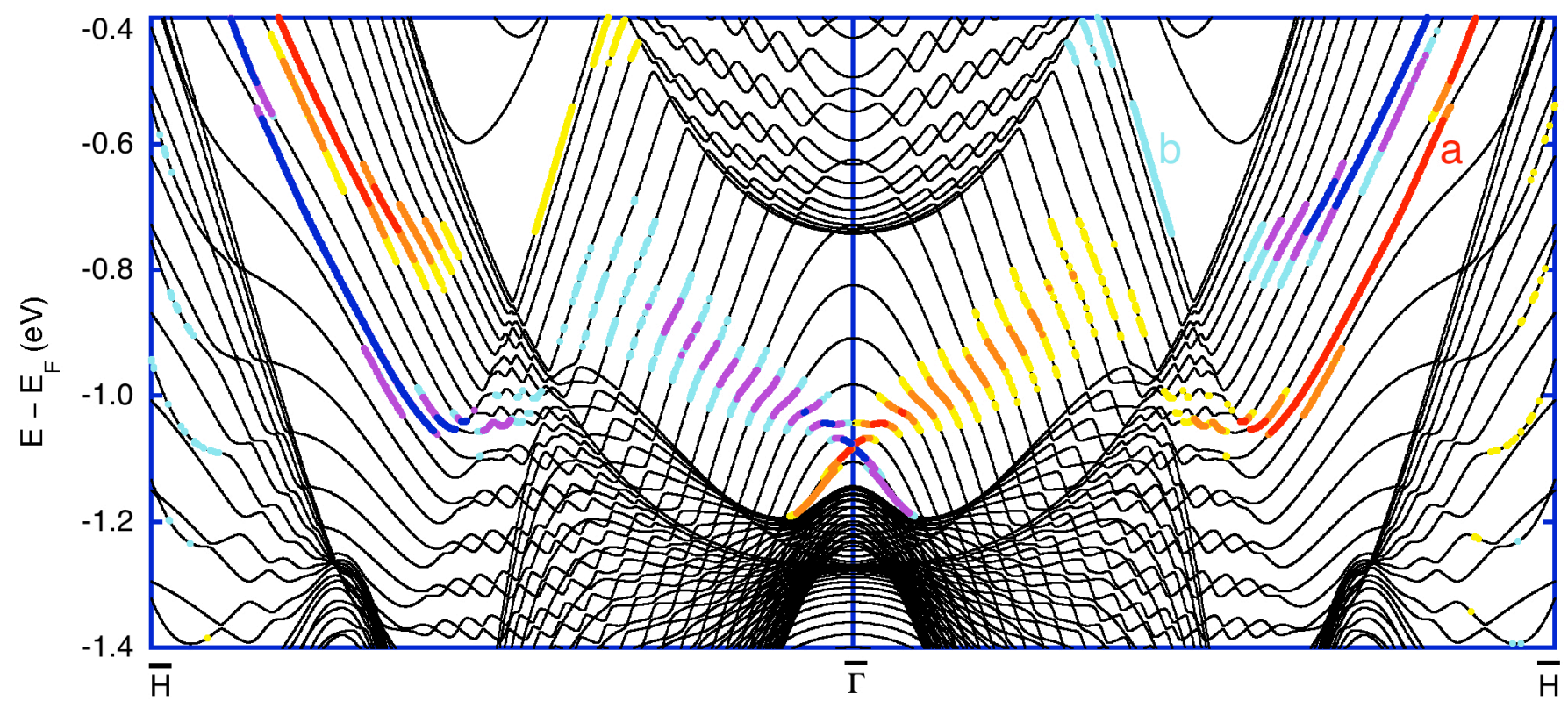

Figure 4. (Color online) Surface states with strong spin polarization (shown in color) in the (110) surface atomic layer of a 50 atomic layer thin film of tungsten with relaxed (110) surfaces calculated with the intersite matrix elements of the spin-orbit Hamiltonian [Eq. (4)] omitted from the calculation. Meaning of the colors is as in Fig. 3.

binding spin-orbit Hamiltonian are primarily responsible for the occurrence of Rashba-like surface states at W(110) surfaces, although the intersite matrix elements modulate some details quantitatively. This finding also strongly suggests that not only intersite matrix elements (as in Ref. 4) but also intrasite matrix elements of the spin-orbit Hamiltonian need to be included when considering Rashba-like effects in theoretical work on topological insulators, spin Hall phenomena and related topics. It also suggests that the mechanism responsible for the Rashba-like dispersion and spin polarization of W(110) surface states differs fundamentally from that of the original Rashba effect since that can be modelled ${ }^{46}$ with just intersite matrix elements of the spin-orbit Hamiltonian. This conclusion is consistent with that reached earlier ${ }^{47}$ (regarding the relative weakness of Rashba splittings due to charge distribution asymmetry) for surface states of other materials, although the reasoning used then ${ }^{47}$ was entirely different and did not involve tight-binding models.

\section{CONCLUSIONS}

In this article, a tight-binding model of bcc tungsten that includes spin-orbit coupling has been introduced and applied to study the properties of the Rashba-Dirac-like surface states of (110) tungsten thin films. Although the model is fitted to only the bulk electronic band structure of bcc tungsten, because it is based on extended Hückel theory it is flexible enough to account very well for the striking effect on the electronic surface states of the films of the relaxation of the surface atomic layer of the tungsten towards the bulk. The results obtained are consistent with the findings of previous $a b$ initio calcula- tions and experiments on the surface states of bulk tungsten crystals. However, the present work has revealed that hybridization with bulk modes impacts the states of the upper and lower Rashba-Dirac cones of W(110) surfaces in qualitatively different ways: While hybridization does not, for the most part, affect the direction of the group velocity of electrons in states belonging to the lower Rashba-Dirac cone, the group velocities of electrons in the states of the upper RashbaDirac cone are reversed by hybridization relative to their direction predicted by Rashba phenomenology, except in the immediate vicinity of the cone's apex. This implies reversal of the directions of travel of spin $\uparrow$ and $\downarrow$ surface electrons of the upper Rashba-Dirac cone relative to those expected from the phenomenology. In this article, the hybridization has also been shown to be much weaker for the lower Rashba-Dirac cone than for the upper Rashba-Dirac cone. The present work has also shown that electric fields that are due to charge transfer between the tungsten and the vacuum near the surface or between the bulk and surface layers do not significantly affect the tungsten (110) Rashba-Dirac surface states. It has also shown the effect of the surface relaxation on the RashbaDirac surface states to be due to the increased overlaps between atomic orbitals of the surface and neighboring layers resulting from the relaxation. It has also demonstrated that, unlike in tight-binding models of the Rashba effect in semiconductor 2DEG's, intrasite (not intersite) matrix elements of the spin-orbit Hamiltonian are primarily responsible for the formation of the tungsten (110) Rashba-Dirac cones, and for their dispersion and their spin polarization. This finding may have important implications for theoretical modeling of topological insulators, spin Hall phenomena and related topics.

This research was supported by NSERC, CIFAR, Westgrid, 
and Compute Canada.

1 Yu. A. Bychkov and E. I. Rashba, Properties of a 2D electron gas with lifted spectral degeneracy, JETP Lett. 39, 78 (1984).

2 Yu. A. Bychkov and E. I. Rashba, Oscillatory effects and the magnetic susceptibility of carriers in inversion layers, J. Phys. C 17, 6039 (1984).

3 Kane, C. L., and E. J. Mele, Quantum Spin Hall Effect in Graphene, Phys. Rev. Lett. 95, 226801 (2005).

4 Kane, C. L., and E. J. Mele, $Z_{2}$ Topological Order and the Quantum Spin Hall Effect, Phys. Rev. Lett. 95, 146802 (2005).

5 S. LaShell, B. A. McDougall, and E. Jensen, Spin Splitting of an Au(111) Surface State Band Observed with Angle Resolved Photoelectron Spectroscopy, Phys. Rev. Lett. 77, 3419 (1996).

${ }^{6}$ G. Nicolay, F. Reinert, S. Hüfner, and P. Blaha, Spin-orbit splitting of the $L$-gap surface state on $\mathrm{Au}(111)$ and $\mathrm{Ag}(111)$, Phys. Rev. B 65, 033407 (2001).

7 M. Hoesch, M. Muntwiler, V. N. Petrov, M. Hengsberger, L. Patthey, M. Shi, M. Falub, T. Greber, and J. Osterwalder, Spin structure of the Shockley surface state on Au(111), Phys. Rev. B 69, 241401(R) (2004).

8 S. N. P. Wissing,C. Eibl, A. Zumbülte, A. B. Schmidt, J. Braun, J. Minàr, H. Ebert, and M. Donath, Rashba-type spin splitting at $\mathrm{Au}(111)$ beyond the Fermi level: the other part of the story, New J. Phys. 15, 105001(2013).

9 R. Requist, P. M. Sheverdyaeva, P. Moras, S. K. Mahatha, C. Carbone, and E. Tosatti, Spin-orbit interaction and Dirac cones in $d$-orbital noble metal surface states, Phys. Rev. B 91, 045432 (2015).

10 E. Rotenberg and S. D. Kevan, Evolution of Fermi Level Crossings versus H Coverage onW(110), Phys. Rev. Lett. 80, 2905 (1998).

11 M. Hochstrasser, J.G. Tobin, E. Rotenberg, and S. D. Kevan, SpinResolved Photoemission of Surface States of W(110)- $(1 \times 1)$ H, Phys. Rev. Lett. 89, 216802 (2002).

12 A. M. Shikin, A. Varykhalov, G.V. Prudnikova, D. Usachov, V.K. Adamchuk, Y. Yamada, J. D. Riley, and O. Rader, Origin of Spin-Orbit Splitting for Monolayers of Au and Ag on W(110) and Mo(110), Phys. Rev. Lett. 100, 057601 (2008).

13 K. Miyamoto, A. Kimura, K. Kuroda, T. Okuda, K. Shimada, H. Namatame, M. Taniguchi, and M. Donath, Spin-Polarized Dirac-Cone-Like Surface State with $d$ Character at W(110), Phys. Rev. Lett. 108, 066808 (2012).

14 K. Miyamoto, A. Kimura, T. Okuda, K. Shimada, H. Iwasawa, H. Hayashi, H. Namatame, M. Taniguchi, and M. Donath, Massless or heavy due to two-fold symmetry: Surface-state electrons at W(110), Phys. Rev. B 86, 161411(R) (2012).

15 A. G. Rybkin, E. E. Krasovskii, D. Marchenko, E. V. Chulkov, A. Varykhalov, O. Rader, and A. M. Shikin, Topology of spin polarization of the $5 d$ states on W(110) and Al/W(110) surfaces, Phys. Rev. B 86, 035117 (2012).

16 D. N. Biswas, P. S. Mandal, S. R. Varier, N. Sahadev and K. Maiti, Evidence of Unusual Spin Polarization of the Surface States of W(110) Surface, SOLID STATE PHYSICS: Proceedings of the 57th DAE Solid State Physics Symposium 2012, AIP Conf. Proc. 1512, 816-817 (2013).

17 H. Mirhosseini, M. Flieger and J. Henk, Dirac-cone-like surface state in $\mathrm{W}(110)$ : dispersion, spin texture and photoemission from first principles New J. Phys. 15, 033019 (2013).

18 F. Giebels, H. Gollisch, and R. Feder, Electron pair emission from W(110): Response to a spin-polarized surface state, Phys. Rev. B 87, 035124 (2013).

19 H. Mirhosseini, F. Giebels, H. Gollisch, J. Henk and R. Feder, Ab initio spin-resolved photoemission and electron pair emission from a Dirac-type surface state in W(110) New J. Phys. 15, 095017 (2013).

20 J. Braun, K. Miyamoto, A. Kimura, T. Okuda, M. Donath, H. Ebert and J. Minàr, Exceptional behavior of $d$-like surface resonances on $\mathrm{W}(110)$ : the one-step model in its density matrix formulation, New J. Phys. 16, 015005 (2014).

21 K. Miyamoto, A. Kimura, T. Okuda and M. Donath, Spin polarization of surface states on W(1 10$)$ : Combined influence of spin-orbit interaction and hybridization, Journal of Electron Spectroscopy and Related Phenomena 201, 53 (2015).
${ }^{22}$ H. Wortelen, H. Mirhosseini, K. Miyamoto, A. B. Schmidt, J. Henk, and M. Donath, Tuning the spin signal from a highly symmetric unpolarized electronic state, Phys. Rev. B 91, 115420 (2015).

23 K. Miyamoto, H.Wortelen, H. Mirhosseini, T. Okuda, A. Kimura, H. Iwasawa, K. Shimada, J. Henk, and M. Donath, Orbital-symmetry-selective spin characterization of Dirac-cone-like state on W(110), Phys. Rev. B 93, 161403(R) (2016).

24 A. Tamai, W. Meevasana, P. D. C. King, C. W. Nicholson, A. de la Torre, E. Rozbicki, and F. Baumberger,Spin-orbit splitting of the Shockley surface state on $\mathrm{Cu}(111)$, Phys. Rev. B 87, 075113 (2013).

25 J. Jiang, S. S. Tsirkin, K. Shimada, H. Iwasawa, M. Arita, H. Anzai, H. Namatame, M. Taniguchi, I. Yu. Sklyadneva, R. Heid, K.-P. Bohnen, P. M. Echenique, and E. V. Chulkov, Many-body interactions and Rashba splitting of the surface state on $\mathrm{Cu}(110)$, Phys. Rev. B 89, 085404 (2014).

26 A. Tanaka, M. Hatano, K. Takahashi, H. Sasaki, S. Suzuki, and S. Sato, Bulk and surface electronic structures of the semimetal Bi studied by angle-resolved photoemission spectroscopy, Phys. Rev. B 59, 1786 (1999).

27 C. R. Ast and H. Höchst, Fermi Surface of Bi(111) Measured by Photoemission Spectroscopy, Phys. Rev. Lett. 87, 177602 (2001).

28 S. Agergaard, Ch. Søndergaard, H. Li, M. B. Nielsen, S. V. Hoffmann, Z. $\mathrm{Li}$ and $\mathrm{Ph}$. Hofmann, The effect of reduced dimensionality on a semimetal: the electronic structure of the Bi(110) surface, New J. Phys. 3, 15 (2001).

29 Yu. M. Koroteev, G. Bihlmayer, J. E. Gayone, E. V. Chulkov, S. Blgel, P. M. Echenique, and Ph. Hofmann, Strong Spin-Orbit Splitting on Bi Surfaces, Phys. Rev. Lett. 93, 046403 (2004).

30 T. Hirahara, K. Miyamoto, I. Matsuda, T. Kadono, A. Kimura, T. Nagao, G. Bihlmayer, E. V. Chulkov, S. Qiao, K. Shimada, H. Namatame, M. Taniguchi, and S. Hasegawa, Direct observation of spin splitting in bismuth surface states, Phys. Rev. B 76, 153305 (2007).

31 A. Kimura, E. E. Krasovskii, R. Nishimura, K. Miyamoto, T. Kadono, K. Kanomaru, E. V. Chulkov, G. Bihlmayer, K. Shimada, H. Namatame, and M. Taniguchi, Strong Rashba-Type Spin Polarization of the Photocurrent from Bulk Continuum States: Experiment and Theory for Bi(111), Phys. Rev. Lett. 105, 076804 (2010)

32 K. Sugawara, T. Sato, S. Souma, T. Takahashi, M. Arai, and T. Sasaki, Fermi Surface and Anisotropic Spin-Orbit Coupling of Sb(111) Studied by Angle-Resolved Photoemission Spectroscopy, Phys. Rev. Lett. 96, 046411 (2006).

33 A. Varykhalov, D. Marchenko, M. R. Scholz, E. D. L. Rienks, T. K. Kim, G. Bihlmayer, J. Sánchez-Barriga, and O. Rader, Ir(111) Surface State with Giant Rashba Splitting Persists under Graphene in Air, Phys. Rev. Lett. 108, 066804 (2012).

34 Sz. Vajna, E. Simon, A. Szilva, K. Palotas, B. Ujfalussy, and L. Szunyogh, Higher-order contributions to the Rashba-Bychkov effect with application to the Bi/Ag(111) surface alloy, Phys. Rev. B 85, 075404 (2012).

35 M. Wolfsberg and L. Helmholz, The Spectra and Electronic Structure of the Tetrahedral Ions $\mathrm{MnO}_{4}^{-}, \mathrm{CrO}_{4}^{--}$, and $\mathrm{ClO}_{4}^{-}$, J. Chem. Phys. 20, 837 (1952).

36 R. Hoffmann, An Extended Huckel Theory. I. Hydrocarbons, J. Chem. Phys. 39, 1397 (1963).

37 J. H. Ammeter, H.-B. Bürgi, J. C. Thibeault, and R. Hoffman, Counterintuitive Orbital Mixing in Semiempirical and ab Initio Molecular Orbital Calculations, J. Am. Chem. Soc. 100, 3686 (1978).

38 For a recent discussion see G. Kirczenow, Molecular nanowires and their properties as electrical conductors, The Oxford Handbook of Nanoscience and Technology,Volume I: Basic Aspects, Chapter 4, edited by A. V. Narlikar and Y. Y. Fu, Oxford University Press, Oxford, U.K. (2010).

39 F. Rostamzadeh Renani and G. Kirczenow, Ligand-Based Transport Resonances of Single-Molecule-Magnet Spin Filters: Suppression of Coulomb Blockade and Determination of Easy-Axis Orientation, Phys. Rev. B 84, 180408(R) (2011).

40 F. Rostamzadeh Renani and G. Kirczenow, Tight Binding Model of Mn12 Single Molecule Magnets: Electronic and Magnetic Structure and Trans- 
port Properties, Phys. Rev. B 85, 245415 (2012)

41 N. E. Christensen and B. Feuerbacher, Volume and surface photoemission from tungsten. I. Calculation of band structure and emission spectra, Phys. Rev. B 10, 2349 (1974).

42 K. Glantschnig and C. Ambrosch-Draxl, Relativistic effects on the linear optical properties of Au, Pt, Pb and W, New J. Phys. 12, 103048 (2010).

43 D.A. Papaconstantopoulos, Handbook of the Band Structure of Elemental Solids, Plenum Press, New York, 1986.

44 D. Venus, S. Cool, M. Plihal, Quantitative structural determination using spin-polarized low-energy electron diffraction rotation curves: W(110), Surf. Sci. 446, 199 (2000)

45 H.L. Meyerheim, D. Sander, R. Popescu, P. Steadman, S. Ferrer, J. Kirschner, Interlayer relaxation of W(110) studied by surface X-ray diffraction, Surf. Sci. 475, 103 (2001).
46 F. Mireles and G. Kirczenow, Ballistic spin-polarized transport and Rashba spin precession in semiconductor nanowires, Phys. Rev. B 64, 024426 (2001)

47 E. E. Krasovskii, Microscopic origin of the relativistic splitting of surface states, Phys. Rev. B 90, 115434 (2014).

48 The YAEHMOP numerical package by G. A. Landrum and W. V. Glassey (Source-Forge, Fremont, California, 2001) was used to compute the overlap matrix $O_{i j}$.

49 S. Konschuh, M. Gmitra, and J. Fabian, Tight-binding theory of the spinorbit coupling in graphene, Phys. Rev. B 82, 245412 (2010).

50 S. Datta and B. Das, Electronic analog of the electro-optic modulator, Appl. Phys. Lett. 56, 665 (1990). 